\title{
The Specificity of Trypanosomal Antigens by Immunofluorescence
}

\author{
BY B. WEITZ \\ Lister Institute of Preventive Medicine, Elstree, Hertfordshire
}

(Received 2 January 1963)

\begin{abstract}
SUMMARY
The immunofluorescent method was applied to a strain of Trypanosoma bruce $i$ and of Trypanosoma vivax by using the direct antibody technique. Conjugated antibodies to the soluble antigens of trypanosomes reacted specifically with the homologous species only; antibodies to the bound antigens reacted with both species.
\end{abstract}

\section{IN'TRODUCTION}

Serological investigations of trypanosomes are considerably limited by the relatively small amounts of antigens available for test. In addition, the heterogeneity of populations of organisms or of antigens derived from them may remain undetected unless the tests are specifically designed to characterize the antigens of individual organisms. The use of the fluorescent labelled antibody technique, therefore, seemed particularly appropriate for the study of the immunological behaviour of trypanosomes, because the precise location of the antigens can be determined on individual organisms as they occur in the blood of infected animals. Fife \& Muschel (1959) described a diagnostic test for antibody to Trypanosoma cruzi in human sera, treating suspensions of formalin-killed $T$. cruzi obtained from cultures with human sera and subsequently with anti-human fluorescent rabbit antibody. In the present work the antigenic character of trypanosomes was studied by allowing specified fluorescent conjugated antisera to react directly with the trypanosomes.

\section{METHODS}

Organisms. Two species of trypanosomes were used: (1) Trypanosoma brucei 427 , a strain isolated from a cow in East Africa; (2) T. vivax, a strain originally isolated from a sheep in West Africa and later adapted to rats and mice (Desowitz \& Watson, 1951, 1953). Both strains were stored at $-70^{\circ}$ in glycerinated suspension in capillaries (Cunningham \& Vickerman, 1962) and when required for tests were maintained in mice by rapid passage.

Antisera. Antisera to the exoantigens (anti-EX) and to the bound antigens (anti-TH) of both species of trypanosomes were obtained from white rats after multiple intramuscular injections of the antigens mixed with potash alum solution as adjuvant (Weitz, 1960). Groups of immunized rats were bled from the heart about 5-10 days after the last injection of antigen and the sera from the bleedings of each group of rats were pooled and stored at $-15^{\circ}$ until required.

Fractionation of antisera. After testing for potency and quality by precipitin 
test (Weitz, 1960, 1962a), the antisera were fractionated by chromatography on DEAE (diethylaminoethyl)-cellulose. The sera were dialysed against $0.01 \mathrm{M}$ phosphate buffer ( $\mathrm{pH} \mathrm{7.5)}$ before fractionation and the eluates concentrated by pressure dialysis to a content of $2.5 \%$ protein and again dialysed. Analysis by starch gel electrophoresis indicated that the fractions used for conjugation contained only the $\beta$ - and $\gamma$-globulin components of rat serum.

Conjugation of globulin fractions. Volumes containing $2.5 \%$ protein ranging from 1-5 ml. were coupled with fluorescent dyes by the method described by Riggs et al. (1958) with the modifications detailed by Goldstein, Slizys \& Chase (1961). The solutions maintained in an ice bath and constantly stirred with a magnetic stirrer were adjusted to $\mathrm{pH} \mathbf{9 \cdot 0 - 9 \cdot 2}$ with cold $\mathrm{M}$-carbonate bicarbonate solution. Crystalline fluorescein isothiocyanate (FICT)* or tetramethylrhodamine isothiocyanate (RICT)* were added slowly at the rate of $0.025 \mathrm{mg} . / \mathrm{mg}$. protein. The coupling was allowed to occur for $1 \mathrm{hr}$. with FICT and for 4-6 hr. with RICT (see Smith, Carski \& Griffin, 1962). At the end of this period the solutions of conjugates were adjusted to $\mathrm{pH} 7 \cdot 2-7 \cdot 6$ with $\mathrm{N}-\mathrm{HCl}$.

Removal of unconjugated fluorescent material. The conjugates were dialysed in the cold overnight against a solution of $0.01 \mathrm{M}$-phosphate buffer in saline ( $\mathrm{pH} \mathrm{7.2)}$ and were then passed through a Sephadex-G25 column, taking only the first dyed effluent fraction. A half volume of acetone-dried rabbit liver as a fine powder was added and shaken mechanically for 18-24 hr. in the cold. The liver powder was then centrifuged down and the clear supernatant fluid removed. Some loss of protein was noted on some occasions; all the conjugates were restored to a final concentration of $2.5 \%$ protein and stored at $-15^{\circ}$.

Preparation of smears. Mice or rats, infected for 2-3 days and containing large numbers of trypanosomes in the blood, were stunned and blood obtained from the sectioned axilla and immediately mixed in 4-5 volumes of Alsever solution (dextrose 4.66 g., sodium chloride 1.05 g., sodium citrate 2.0 g., distilled water $200 \mathrm{ml}$., pH 6.1). Blood smears on microscope slides were prepared as soon as practicable after the mixing in Alsever's solution and were allowed to dry in air. As soon as the slides were dry they were fixed in methanol for 1 min., shaken dry and dried in air on a warm plate.

Staining of blood smears. Comparisons between different treatments of the same preparation were all made on the same slide on which several small areas were separated by painting with nail varnish, a procedure which prevented the different reagents from mixing with each other, and which allowed the microscopic examination of the differently treated areas of one slide without altering the illumination, thus ensuring a correct assessment of the different intensities of staining or fluorescence.

The fluorescent antibody, used neat or diluted in phosphate buffered saline, was applied to the appropriate section of the slide, covering the whole area of the section. After $10 \mathrm{~min}$. at room temperature, the conjugate was removed by suction with a small capillary pipette and the sections washed with phosphate buffered saline, first by carefully squirting each section with a pipette and then by covering the whole slide for $1 \mathrm{~min}$; the buffer solution was then shaken off and the smears dried

* From the Baltimore Biological Laboratory, Baltimore 18, Maryland, U.S.A. 
in air on a warm plate. In this way the possibility of contaminating sections with the different reagents during washing was minimized.

Examination of specimens. A Reichert fluorescence illuminator was used, consisting of an Osram HBO-200 W. mercury vapour lamp, with adjustable condenser and field diaphragm. The smears were examined in a darkened room with darkground illumination; a control smear stained with acridine orange was used to adjust the apparatus initially. Various primary filters were used, singly or combined, including BG 12/3 mm., UG 1/1.5 mm. and UG 1/2.5 mm. (Schott \& Genossen) and various ocular barrier filter combinations, including a Wratten $2 \mathrm{~B}$, pale greenyellow filter and orange-yellow filter OG 1/1.5 mm., all with ultraviolet-stopping glass GG 9/1 mm.

Owing to the very rapid loss of fluorescence of the smears under illumination, photographs were taken with a fast $35 \mathrm{~mm}$. Kodak TRI-X film (ASA 400) giving about 3-4 min. exposures with a BG $12 / 3 \mathrm{~mm}$. primary filter and UG 1 barrier filter under $1 / 12$ in. oil-immersion objective and $\times 6$ ocular. Colour transparencies were obtained with Kodak high-speed daylight Ektachrome film (ASA 160) by using the same objectives and ocular with a UG $1 / 2.5 \mathrm{~mm}$. or UG $1 / 1.5 \mathrm{~mm}$. primary filter and a pale green-yellow barrier filter and allowing $10 \mathrm{~min}$. exposures.

\section{RESULTS AND DISCUSSION}

Non-specific fluorescence of trypanosomes. Many preparations of trypanosomes showed non-specific fluorescence of various intensities, and no results were accepted unless control preparations fluoresced either not at all, or only minimally. The controls consisted of portions of the smears left untreated and portions which had been treated with conjugated antisera to rabbit serum protein, which should not react with the preparations derived from mouse or rat. Other controls were treated with normal rat serum or normal rabbit sera similarly conjugated with fluorescent dyes. Unless the control preparations showed no more than a low-intensity fluorescence, which appeared unavoidable under the conditions of the test, the results were rejected.

No single factor proved to be mainly responsible for the production of nonspecific fluorescence of trypanosomes. The best results were obtained when the diluted infected blood was smeared without delay and when the smears were fixed immediately after the films were dry. Suspensions of trypanosomes obtained by centrifugation usually yielded non-specifically stained specimens, and thus smears of diluted whole blood were mostly used. Washing the preparations for prolonged periods was found unnecessary and gave poor results. It thus seemed that any step which favoured the proper preservation of the trypanosomes was useful in avoiding non-specific fluorescence.

Specific fluorescence. The results of treating blood smears of Trypanosoma brucei and T. vivax with anti-EX and anti-TH are illustrated in Pl. 1, figs. 1-8. The specificity of the anti-exoantigen is shown by the well-marked staining of homologous species only (Pl. 1, figs. 1, 2, 5, 6). The anti-TH sera on the other hand stained the smears of both species equally (Pl. 1, figs. 3, 4, 7, 8). These findings were confirmed by making mixed smears of the two species and treating them with antisera conjugated with different dyes. When a mixture of $T$. brucei and $T$. vivax was treated 
with 'brucei' anti-EX conjugated with FITC and subsequently with 'vivax' anti-EX conjugated with RITC, the respective organisms were stained green and red, showing that only the homologous species was stained by the homologous antiserum. When the mixed organisms were similarly treated with two differently conjugated anti-TH of different colour, both species of trypanosomes appeared greenish yellow as a result of the double staining with fluorescein and rhodamine. No difference was noted in the localization of the antigens with the different antisera.

These results are consistent with the properties of exoantigen as determined by other methods. Thus, mice immunized with the exoantigen of one species are protected against infection with the homologous species but not with a heterologous species (Weitz, 1962b). Similarly, anti-exoantigen sera precipitate only the homologous exoantigen, whereas anti-TH precipitating sera cross-react with the heterologous species (Weitz, 1962a). The results confirm the distinctive characters of the soluble antigens found in the sera of infected animals (exoantigen) and those which are derived from the disruption of the trypanosome (bound antigens). The exoantigens determine the small differences in antigenic structure of different species of trypanosome. Moreover, antigenic variation appears to affect the exoantigen more than other antigenic components in the organisms. For immunization of animals against infections with trypanosomes it is evident that the bound antigens are therefore more likely to promote antibodies effective against more species of trypanosomes and possibly their variants than the antibodies provoked solely by exoantigens. Further investigations are being made to confirm these results with a variety of strains and species of trypanosomes.

\section{REFERENCES}

Cunningham, M. P. \& Vickerman, K. (1962). Antigenic analysis in the Trypanosoma brucei group, using the agglutination reaction. Trans. R. Soc. trop. Med. Hyg. 56, 48.

Desowitz, R. S. \& Watson, H. J. C. (1951). Studies on Trypanosoma vivax. I. Susceptibility of white rats to infection. Ann. trop. Med. Parasit. 45, 207.

Desowitz, R.S. \& Watson, H. J. C. (1953). Studies on Trypanosoma vivax. IV. The maintenance of a strain in white rats without sheep-serum supplement. Ann. trop. Med. Parasit. 47, 62.

Fife, E. H. \& Muschel, L. H. (1959). Fluorescent antibody technic for serodiagnosis of Trypanosoma cruzi infection. Proc. Soc. exp. Biol., N.Y. 101, 540.

Goldstein, G., Slizys, I. S. \& Chase, M. W. (1961). Studies on fluorescent antibody staining. I. Non-specific fluorescence with fluorescein-coupled sheep anti-rabbit globulins. J. exp. Med. 114, 89.

Riggs, R. J., Seiwald, J. H., Burckhalter, C. M., Downs, T. G. \& Metcalf, T. G. (1958). Isothiocyanate compounds as fluorescent labeling agents for immune sera. Amer. J. Path. 34, 1081.

Smith, M. L., Carski, T. R. \& Griffin, C. W. (1962). Modification of fluorescent antibody procedures when employing crystalline tetramethylrhodamine isothiocyanate. J. Bact. 83, 1358.

WEITZ, B. (1960). The properties of some antigens of Trypanosoma brucei. J. gen. Microbiol. 23, 589 .

Weitz, B. (1962a). Immunity in Trypanosomiasis. In Drugs, Parasites and Hosts. Ed. by L. G. Goodwin and R. H. Nimmo-Smith. Biol. Counc. Symp. London: Churchill.

WerTz, B. $(1962 b)$. The antigenicity of some African Trypanosomes. In Immunity to Protozoa. Ed. by P. C. C. Garnham, A. E. Pierce and I. M. Roitt. Symp. Brit. Soc. Immunol. Oxford: Blackwell Scientific Publications. 


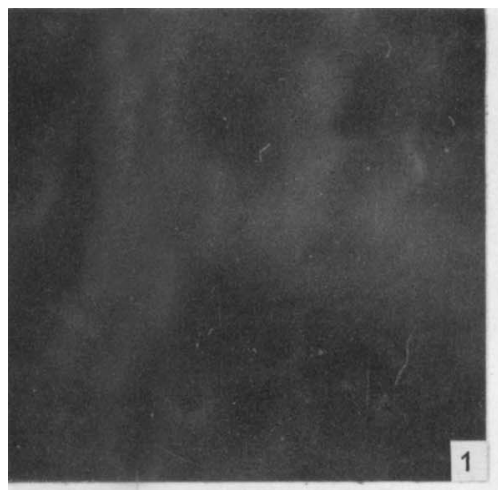

Anti-brucei

exoantigen

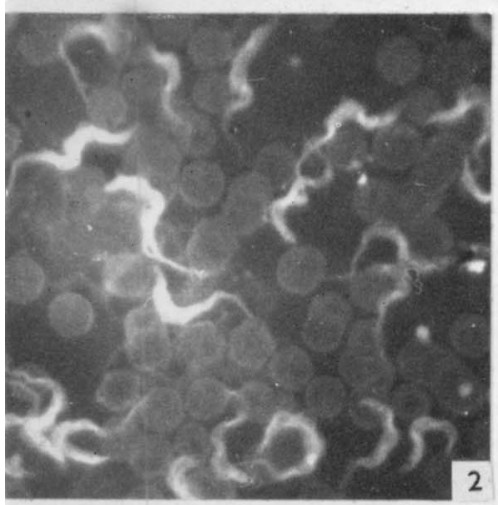

Anti-vivax

exoantigen

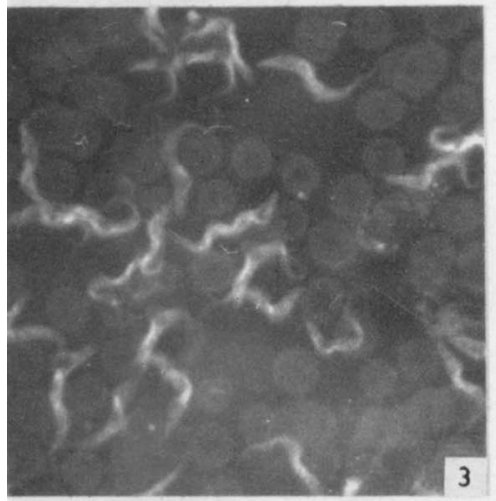

Anti-brucei bound antigen

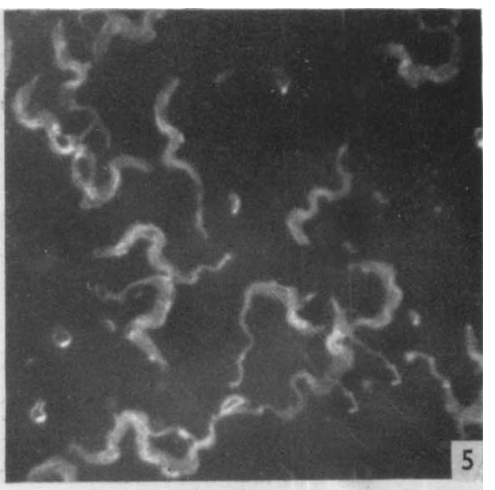

3

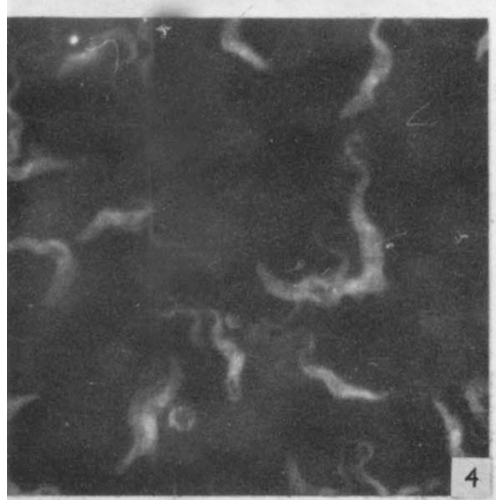

Anti-vivax bound antigen
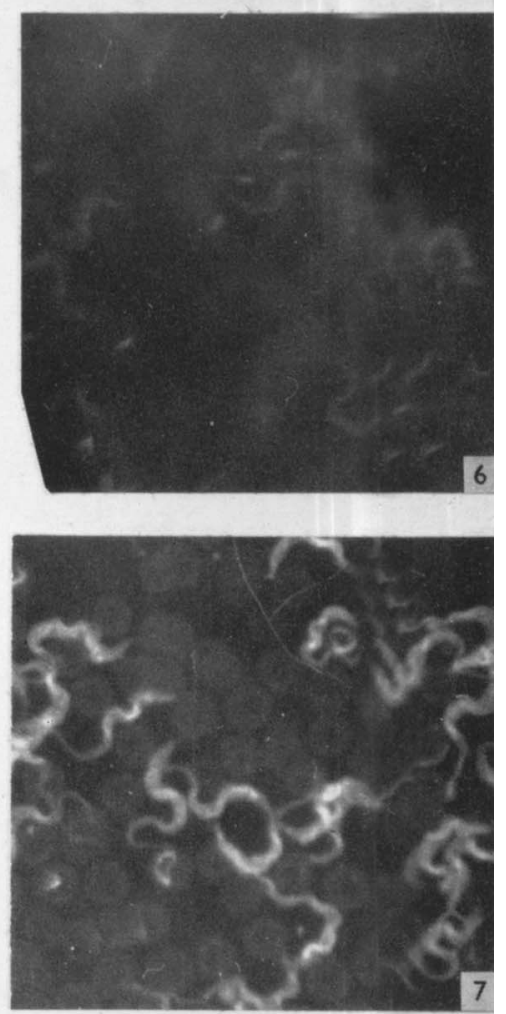

Trypanosoma vivax

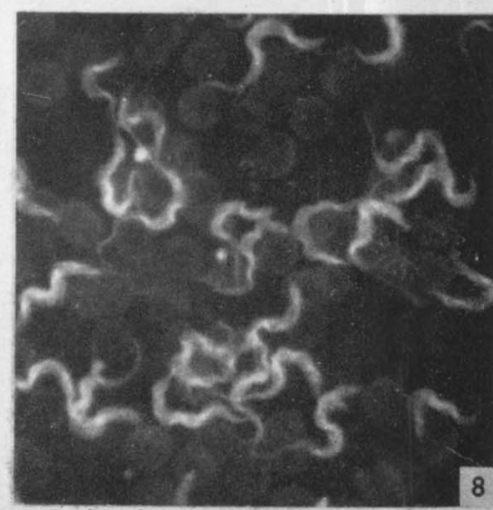

Trypanosoma brucei 


\section{EXPLANATION OF PLATE}

Figs. 1-4 Trypanosoma vivax, Figs. 5-8 Trypanosoma brucei, treated with fluorescent antibody. All photographs were taken with a $3 \mathrm{~min}$. exposure, developed and printed together under the same conditions. The intensities of fluorescence are comparable for each species of trypanosome since the photographs were made from the same slide without alteration of the illumination, with a BG $12 / 3 \mathrm{~mm}$. exciter filter and OG 1 barrier filter and examined under $1 / 12 \mathrm{in}$. oil immersion objective with $a \times 6$ ocular.

Figs. 1, 5. Treated with fluorescent antibody to T. brucei exoantigen (anti-EX).

Figs. 2, 6. Treated with fluorescent antibody to T. vivax exoantigen (anti-EX).

Figs. 3, 7. Treated with fluorescent antibody to T. brucei bound antigens (anti-TH).

Figs. 4, 8. Treated with fluorescent antibody to $T$. vivax bound antigens (anti-TH). 